\title{
Three Cases of Sacral Pressure Ulcers Presenting Primary Dermatoporosis on the Forearms
}

\author{
Yuta Kurashige Tokuya Minemuta Tetsuo Nagatani \\ Department of Dermatology, Hachioji Medical Center, Tokyo Medical University, \\ Tokyo, Japan
}

\section{Key Words}

Aging skin · Chronological aging · Dermatoporosis · Skin fragility · Photoaging ·

Pressure ulcer

\begin{abstract}
The relatively new term dermatoporosis refers to chronic deficiencies in the skin's functions in the elderly population due to aging. This syndrome is marked by chronic cutaneous fragility clinically represented by skin atrophy, senile purpura, stellate pseudoscars, skin laceration, and dissecting hematoma of the skin. In this paper, we report three cases of sacral pressure ulcers presenting primary dermatoporosis on the forearms. Case 1 was a 74-yearold male who presented with a stage IV sacral pressure ulcer. The signs of dermatoporosis appeared on the forearms. Histopathology of the lesions revealed epidermal thinning with loss of rete ridges. Azan and Elastica van Gieson staining demonstrated the degeneration of the dermal collagen fibers and elastic fibers, respectively. In spite of 6 months of treatment, the ulcer failed to heal sufficiently. Case 2 was a 74-year-old male and Case 3 was a 97-yearold female. Both cases presented with a stage II sacral pressure ulcer and dermatoporosis on the forearms. Histopathological examinations and the clinical course of the wound could not be ascertained in Cases 2 and 3. None of the patients had previously used corticosteroids. The presence of a primary dermatoporosis on the forearms in these cases may be associated with the increased risk of pressure ulcer development.
\end{abstract}

\section{Introduction}

The term dermatoporosis, proposed by Kaya and Saurat in 2007 [1], refers to the chronic cutaneous insufficiency and fragility due to aging. This condition can start from the age of 
60 years and be fully developed between 70 and 90 years. As summarized in table 1, typical clinical manifestations of dermatoporosis are skin atrophy, senile purpura, stellate pseudoscars, skin laceration, and dissecting hematoma of the skin. Moreover, dermatoporosis can be classified into primary and secondary lesions. The former is related to chronological aging and photoaging, the result of long-term sun exposure. By contrast, the latter is associated with chronic use of topical or systemic corticosteroids. While delayed cutaneous wound healing has been mentioned as one of the clinical features of this condition [1], the connection between dermatoporosis and the development of pressure ulcers has rarely been made in previous English-language publications pertaining to this syndrome [1-6]. Herein, we report three cases of sacral pressure ulcers presenting primary dermatoporosis on the forearms.

\section{Case Report}

Case 1

A 74-year-old male, who was afflicted with Parkinson's disease, was admitted to our hospital for treatment of a stage IV sacral pressure ulcer (National Pressure Ulcer Advisory Panel, 1989 [7]). This lesion measured $3 \times 4 \mathrm{~cm}$ and included a large undermining skin pocket (fig. 1a). In addition, senile purpura, stellate pseudoscars, and skin lacerations were observed on the forearms (fig. 1b). These findings were consistent with a stage II primary dermatoporosis [1]. The patient had not previously used, or been administered, corticosteroids. Histopathology of the left forearm revealed epidermal thinning with loss of the normal rete ridge pattern and dermal solar elastosis (fig. 1c). Azan and Elastica van Gieson staining demonstrated the degeneration of the dermal collagen fibers and elastic fibers, respectively (fig. 1d, e). After 6 months of treatment, the patient was transferred to an extended care hospital, although even at this point the wound had not healed sufficiently (fig. 1f).

Case 2

A 74-year-old male who had been admitted to the coronary care unit of our hospital due to serious heart failure developed a stage II sacral pressure ulcer that measured $1.5 \times 3 \mathrm{~cm}$ (fig. 2a). On the forearms were clinical signs of stage II primary dermatoporosis, including skin atrophy, senile purpura, stellate pseudoscars, and skin lacerations (fig. $2 \mathrm{~b}$ ). The patient had not previously used, or been administered, corticosteroids. We could not perform a histopathological examination or conduct a long-term follow-up of the patient's condition because of his death 2 weeks after consultation.

Case 3

A 97-year-old female with gait disorder visited our outpatient clinic for treatment of a stage II sacral pressure ulcer that measured $2 \times 2.5 \mathrm{~cm}$ (fig. 3a). On her forearms were clinical signs of stage I primary dermatoporosis, such as skin atrophy, senile purpura, and stellate pseudoscars (fig. 3b). She, too, had never previously used corticosteroids. We could not conduct a histopathological examination or monitor the clinical course of the wound because the patient failed to attend her outpatient appointments. 


\section{Discussion}

Aging skin, weakened by a combination of photoaging and chronological aging [8], may present decreased tolerance for friction and shear forces. Accordingly, both photoaging and chronological aging have been mentioned as risk factors of pressure ulcer development [810]. In addition, both types of aging skin may contribute to chronic skin fragility known as dermatoporosis. This syndrome is comprised of some structural abnormalities that include (1) modifications of the extracellular matrix such as collagen fibers and elastic fibers, and (2) alterations in skin viscoelasticity [1]. Moreover, histopathology of the characteristic lesions can demonstrate the flattening of rete ridges and solar elastosis (table 1). Such disorders in dermatoporosis are consistent with the previously described features of photoaging and/or chronological aging [8].

In the present cases, the signs of dermatoporosis were restricted to the forearms and were rarely seen around the sacral pressure ulcers, although delayed wound healing was noted in Case 1. On the other hand, the chronological skin aging, a component of primary dermatoporosis, may have spread systemically from the forearms to other areas of the skin. If this is true, besides the comorbidities in each case, such systemic skin aging might also contribute to pressure ulcer development.

Given these considerations, we speculate that dermatoporosis may occur with greater frequency in more patients presenting pressure ulcers, and that the presence of dermatoporosis on the forearms may also be suggestive of an increased risk of pressure ulcer development. To confirm this hypothesis, a comprehensive investigation targeting a larger population is required.

\section{References}

$\$ 1$ Kaya G, Saurat JH: Dermatoporosis: a chronic cutaneous insufficiency/fragility syndrome. Clinicopathological features, mechanisms, prevention and potential treatments. Dermatology 2007;215:284-294.

-2 Saurat JH: Dermatoporosis. The functional side of skin aging. Dermatology 2007;215:271-272.

-3 Kaya G, Jacobs F, Prins C, Viero D, Kaya A, Saurat JH: Deep dissecting hematoma: an emerging severe complication of dermatoporosis. Arch Dermatol 2008;144:1303-1308.

4 Barnes L, Tran C, Sorg O, Hotz R, Grand D, Carraux P, Didierjean L, Stamenkovic I, Saurat JH, Kaya G: Synergistic effect of hyaluronate fragments in retinaldehyde-induced skin hyperplasia which is a Cd44dependent phenomenon. PLoS One 2010;5:e14372.

5 Mengeaud V, Dautezac-Vieu C, Josse G, Vellas B, Schmitt AM: Prevalence of dermatoporosis in elderly French hospital in-patients: a cross-sectional study. Br J Dermatol 2012;166:442-443.

6 Kaya G: New therapeutic targets in dermatoporosis. J Nutr Health Aging 2012;16:285-288.

-7 Pressure ulcers prevalence, cost and risk assessment: consensus development conference statement The National Pressure Ulcer Advisory Panel. Decubitus 1989;2:24-28.

8 Garcia AD, Thomas DR: Assessment and management of chronic pressure ulcers in the elderly. Med Clin North Am 2006;90:925-944.

-9 Levine JM, Simpson M, McDonald RJ: Pressure sores: a plan for primary care prevention. Geriatrics 1989;44:75-90.

10 Witkowski JA, Parish LC: The decubitus ulcer: skin failure and destructive behavior. Int J Dermatol 2000;39:894-896. 
Kurashige et al.: Three Cases of Sacral Pressure Ulcers Presenting Primary

Dermatoporosis on the Forearms

Table 1. Characteristics of respective clinical manifestations and proposed clinical staging of dermatoporosis

\begin{tabular}{|c|c|c|c|c|}
\hline Clinical manifestations & $\begin{array}{l}\text { Stage I } \\
\text { (case 3) }\end{array}$ & $\begin{array}{l}\text { Stage II } \\
\text { (case } 1,2)\end{array}$ & Stage III & Stage IV \\
\hline \multicolumn{5}{|l|}{ Skin Atrophy } \\
\hline Very thin skin with numerous wrinkles & + & + & + & + \\
\hline \multicolumn{5}{|l|}{ Senile purpura } \\
\hline $\begin{array}{l}\text { Repetitive, spontaneous dermal bleeding without coagulation disorder } \\
\text { Stellate pseudoscar }\end{array}$ & + & + & + & + \\
\hline Spontaneous dermal laceration displaying a stellar aspect & + & + & + & + \\
\hline \multicolumn{5}{|l|}{ Skin laceration } \\
\hline Expression of skin fragility resulting from minor traumas & - & $+(<10)$ & $++(\geq 10)$ & $++(\geq 10)$ \\
\hline Dissecting hematoma of the skin & & & & \\
\hline $\begin{array}{l}\text { Subcutaneous massive bleeding with surrounding ischemic necrosis and } \\
\text { erythematous swelling of the superficial skin }\end{array}$ & - & - & - & + \\
\hline
\end{tabular}

Cited and modified from Kaya and Saurat, 2007 [1]. 


\section{Case Reports in Dermatology}

\begin{tabular}{l|l}
\hline Case Rep Dermatol 2013;5:73-78 \\
\hline DOI: $\underline{10.1159 / 000350286}$ & $\begin{array}{l}\text { C 2013 S. Karger AG, Basel } \\
\text { www.karger.com/cde }\end{array}$ \\
\hline
\end{tabular}

Kurashige et al:: Three Cases of Sacral Pressure Ulcers Presenting Primary

Dermatoporosis on the Forearms
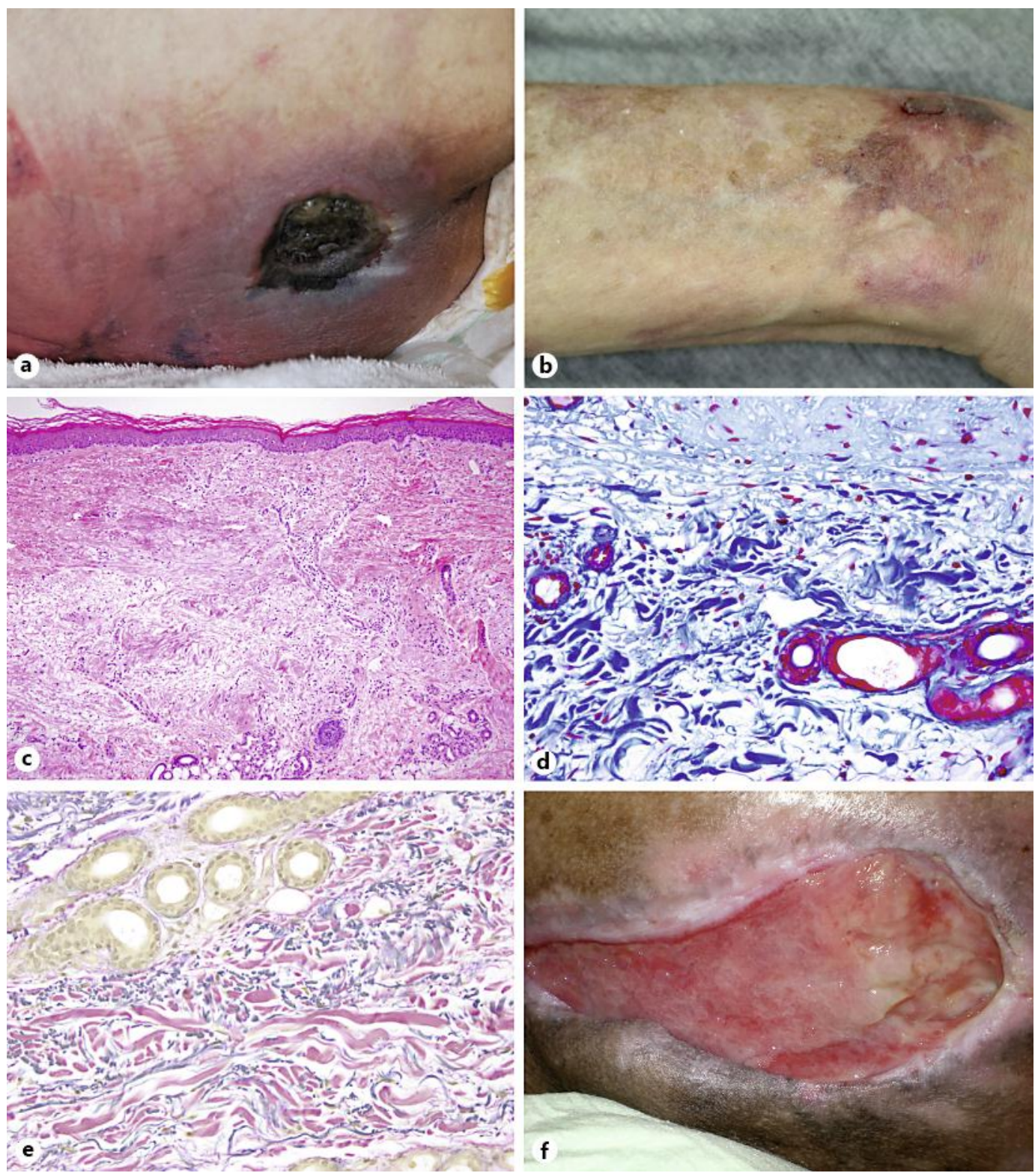

Fig. 1. Clinicopathological findings of Case 1. a Stage IV pressure ulcer with skin pocket was noted in the patient's sacral area. b Skin atrophy, senile purpura, stellate pseudoscars, and skin lacerations were observed on the forearms. c Histopathology revealed epidermal thinning and dermal solar elastosis (Hematoxylin and eosin, $\times 100$ ). $\mathbf{d}$ Azan staining demonstrated the degeneration of dermal collagen fibers, here stained blue $(\times 400)$. e Elastica van Gieson staining demonstrated the degeneration of elastic fibers, here stained black $(\times 400)$. $f$ In spite of treatment including skin incision, the sacral pressure ulcer had still not healed completely 6 months afterwards. 

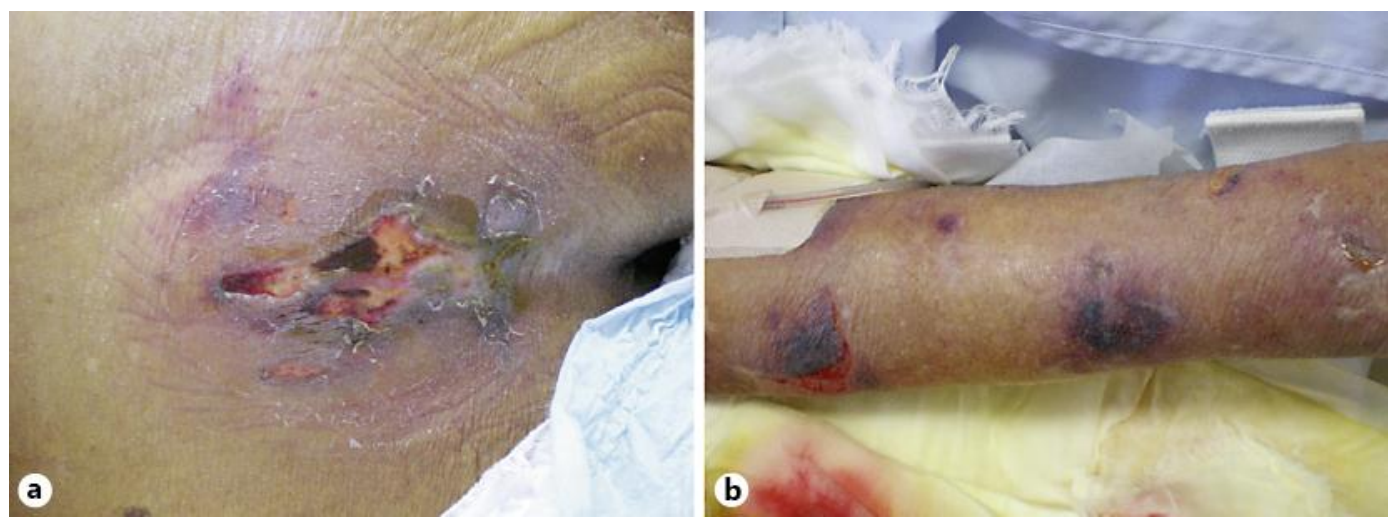

Fig. 2. Clinical appearance of Case 2. a Stage II pressure ulcer developed on the sacral area. b Skin atrophy, senile purpura, stellate pseudoscars, and skin lacerations, were noted on the forearms.
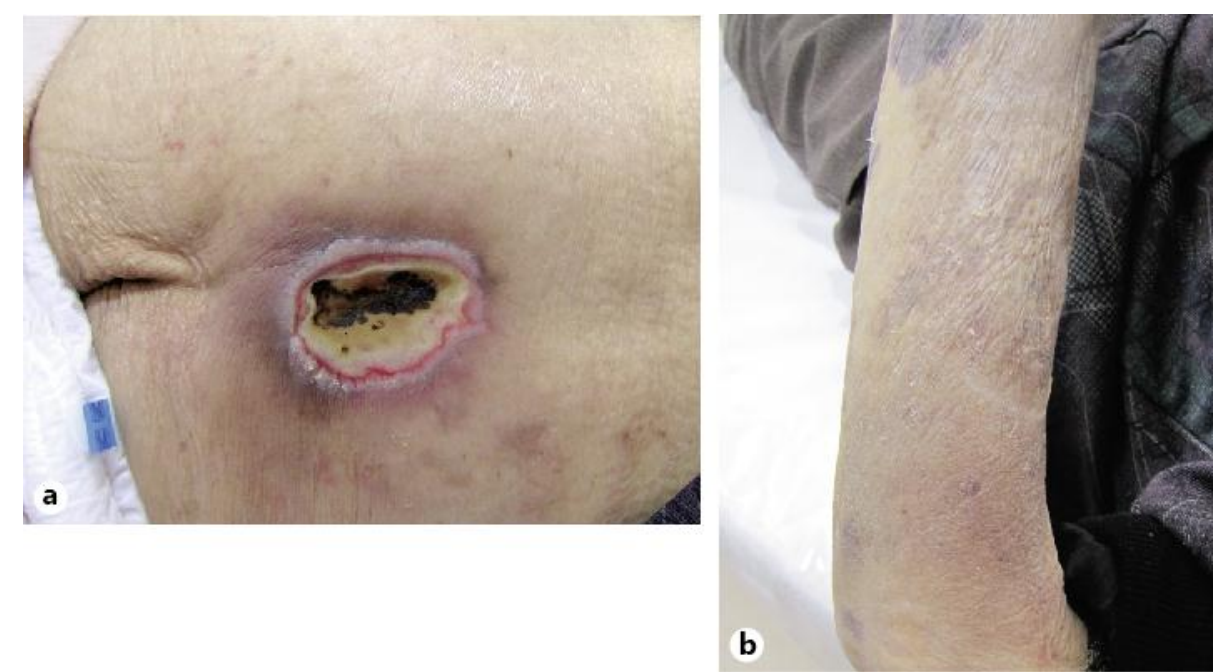

Fig. 3. Clinical appearance of Case 3. a Stage II pressure ulcer developed in the sacral area. b Skin atrophy, senile purpura, and stellate pseudoscars were again noted on the forearms. 